\title{
A note on the existence of CAPM equilibria with homogeneous Cumulative Prospect Theory preferences.
}

\author{
M. Del Vigna*
}

January 12, 2012

\begin{abstract}
This note identifies and fixes a minor gap in Proposition 1 in Barberis and Huang (2008). Assuming homogeneous Cumulative Prospect Theory decision makers, we show that CAPM is a necessary (though not sufficient) condition that must hold in equilibrium. We support our result with numerical examples where security prices become negative.
\end{abstract}

Keywords: asset pricing, capital asset pricing model, cumulative prospect theory. AMS classification (2000): 91B25, 91G10.

JEL classification: C62, D53, G11, G12.

\section{Introduction}

CAPM has been widely recognized as one of the cornerstones of modern finance. While it originated from expected utility theory, in the past few years its robustness progressively came to light. Notably, CAPM is a suitable basis also under different choice paradigms. In an earlier version of their working paper in 2003, De Giorgi, Hens and Levy were the first in proving that CAPM can hold when investors follow Cumulative Prospect Theory (CPT) by Kahneman and Tversky (1992). In a subsequent published article, Barberis and Huang (2008) re-proposed the CAPM with a brand new proof. These authors showed that CPT preferences can even satisfy second-order stochastic dominance when risky asset payoffs have a particular distribution. Furthermore, they shed light on the pricing implications of CPT when skewed securities are introduced in the economy.

The scope of the present note is to fix a minor gap in a result by Barberis and Huang (2008). Briefly, Proposition 1 in the aforementioned paper asserts that in a market with normally distributed payoffs and special homogeneous CPT preferences, a CAPM equilibrium with positive prices always exists. However, we are going to provide numerical examples where negative prices are needed to guarantee the existence of a CAPM equilibrium. Intuitively, negative prices are necessary when agents are excessively loss adverse and/or risk is extremely high. This is because even if a risky asset has zero price, it may happen that no agent is willing to hold it in her portfolio. Consequently, market clearing is not satisfied unless (some) prices become negative. Our critique similarly applies to related results about existence of CAPM equilibrium with heterogeneous CPT investors (see Del Vigna (2011)).

\section{The result}

We recover the setting of Barberis and Huang (2008) and we keep the same notation whenever possible. Let us consider a one-period economy with initial date $t=0$ and terminal date $t=1$.

Assumption 1 (Market structure). There are $J+1$ traded assets in a frictionless market without constraints.

- Asset 0 is the risk-free asset with exogenous gross return $R_{f}$ and it is in perfectly elastic supply.

\footnotetext{
*Université Paris-Dauphine \& Università degli Studi di Pisa, matteo.delvigna@for.unipi.it.
} 
- Assets $1, \ldots, J$ are non-redundant risky securities, whose per-share payoff at time 1 is a multivariate normal r.v. $\tilde{X}=\left(\tilde{X}_{1}, \ldots, \tilde{X}_{J}\right)$, namely $\tilde{X} \sim N(m, V)$ where $m \in \mathbb{R}^{J}$ and $V \in \mathbb{R}^{J \times J}$ is a positive-definite variance-covariance matrix. The exogenous supply of the risky assets is $N=\left(n_{1}, \ldots, n_{J}\right) \in \mathbb{R}_{++}^{J}{ }^{1}$.

We denote $p=\left(p_{1}, \ldots, p_{J}\right)$ the prices of the $J$ stocks to be determined in equilibrium. The returns of the risky securities will be $\left(\tilde{R}_{1}, \ldots, \tilde{R}_{J}\right)$, where $\tilde{R}_{j}=\tilde{X}_{j} / p_{j}$. Portfolios are obtained through investment in the $J+1$ securities and we can consider the market portfolio $\tilde{M}=\tilde{X}^{\top} N$, that is the value of all available assets. The expected payoff, the variance and the price of $\tilde{M}$ will be given by $m_{M}=m^{\top} N, s_{M}^{2}=N^{\top} V N$ and $p_{M}=p^{\top} N$ respectively. Then, $\tilde{R}_{M}=\tilde{M} / p_{M}$ will be the market return.

All the traders we consider follow the CPT paradigm as introduced in Kahneman and Tversky (1992). Specifically, suppose that an investor is endowed with initial wealth $W_{0}$ and obtains the terminal wealth $\tilde{W}=W_{0} \tilde{R}$ through her investment strategy. Then CPT states that the decision maker evaluates deviations of the terminal wealth from a reference wealth level $W_{z}$. In other words, she is interested in gains or losses, namely $\hat{W} \equiv \tilde{W}-W_{z}$. According to Barberis and Huang (2008), we set $W_{z}=W_{0} R_{f}$ so that

$$
\hat{W} \equiv \tilde{W}-W_{0} R_{f}
$$

Following CPT, the goal function of our trader will be

$$
V(\hat{W})=-\int_{0}^{+\infty} v(W) \mathrm{d} w^{+}(1-P(W))+\int_{-\infty}^{0} v(W) \mathrm{d} w^{-}(P(W)),
$$

where $v$ is the value (utility) function, $w^{+}, w^{-}$are the probability weighting functions and $P$ is the cumulative distribution function (cdf) of $\hat{W}$. Barberis and Huang (2008) assume a piecewise-power utility function with identical exponents, that is

$$
v(x)= \begin{cases}x^{\alpha} & \text { if } x \geq 0 \\ -\lambda(-x)^{\alpha} & \text { if } x<0\end{cases}
$$

for some $\alpha \in(0,1)$ and $\lambda>1$. Furthermore, they set

$$
w^{+}(p)=w^{-}(p) \equiv w(p)=\frac{p^{\delta}}{\left(p^{\delta}+(1-p)^{\delta}\right)^{1 / \delta}},
$$

with $\delta \in(0.28,1)$. Note that this specification is very close to the original one by Tversky and Kahneman (1992). $\lambda>1$ models loss aversion whereas $\delta>0.28$ ensures monotonicity of $w$. Experimental evidence suggests $\alpha \approx 0.88, \lambda \approx 2.25, \delta \approx 0.65$.

Assumption 2 (Investor preferences). The economy is populated by a large number of price-taking agents who derive utility from gains or losses $\hat{W}$ given in (1). All investors have the same preferences as specified in equations (2)-(4). In particular, the parameters $\alpha, \lambda$ and $\delta$ are the same for all the traders.

We complete the set of hypotheses with the following

Assumption 3 (Investor endowments and beliefs). Each investor receives a positive initial wealth in the form of traded assets and assigns the same probability distribution to future payoffs and security returns.

Proposition 1 in Barberis and Huang (2008) states that under Assumptions 1-3, there always exists a CAPM equilibrium ${ }^{2}$. In other words, the following linear relations hold

$$
E\left(\tilde{R}_{j}\right)-R_{f}=\beta_{j}\left(E\left(\tilde{R}_{M}\right)-R_{f}\right), \quad j=1, \ldots, J
$$

where

$$
\beta_{j} \equiv \frac{\operatorname{Cov}\left(\tilde{R}_{j}, \tilde{R}_{M}\right)}{\operatorname{Var}\left(\tilde{R}_{M}\right)} .
$$

\footnotetext{
${ }^{1} \mathbb{R}_{++}^{J}$ is short notation for $(0,+\infty)^{J}$. We simply mean that every asset is in strictly positive supply.

${ }^{2}$ The authors do not give a formal definition of equilibrium. However, an equilibrium is a price vector $p \in \mathbb{R}_{++}^{J}$ and portfolios $\Theta$ of the investors such that $(i) \Theta$ are optimal given $p$ and (ii) the market for the risky securities is cleared.
} 
Moreover, the excess market return $\hat{R}_{M} \equiv \tilde{R}_{M}-R_{f}$ satisfies

$$
V\left(\hat{R}_{M}\right) \equiv-\int_{-\infty}^{0} w\left(P\left(\hat{R}_{M}\right)\right) \mathrm{d} v\left(\hat{R}_{M}\right)+\int_{0}^{+\infty} w\left(1-P\left(\hat{R}_{M}\right)\right) \mathrm{d} v\left(\hat{R}_{M}\right)=0
$$

and the market risk premium is positive:

$$
E\left(\hat{R}_{M}\right)>0
$$

Actually, equations (5)-(8) constitute necessary but not sufficient conditions for an equilibrium. Therefore, the correct framing of Proposition 1 in Barberis and Huang (2008) is

Proposition 1. Under Assumptions 1-3, if an equilibrium exists then equations (5)-(8) hold.

For a detailed proof, we refer the reader to the Appendix in Barberis and Huang (2008). We remark that when proving the necessary part of their Proposition 1, the authors assume that all securities have positive prices in equilibrium. Conversely, for the sufficient part they claim that one can compute the equilibrium price vector $p$ using $J-1$ non-redundant equations from (5) and the additional equation (7) which yields the equilibrium market price $p_{M}$. Now, if the resulting $p \in \mathbb{R}_{++}^{J}$, then it will be the equilibrium price vector. Furthermore, the equilibrium holdings can be arbitrarily chosen as long as they clear the market. However, nothing guarantees that such prices are strictly positive.

In Section III.A, Barberis and Huang (2008) provide a numerical example of equilibrium. To begin, they exploit Assumption 1 to set $\hat{R}_{M} \sim N\left(\mu_{M}, \sigma_{M}^{2}\right)$. After that, they specify the parameters as follows: $\left(\alpha, \delta, \lambda, R_{f}, \sigma_{M}\right)=(0.88,0.65,2.25,1.02,0.15)$. Inserting these values into equation (7), they correctly state that $V\left(\hat{R}_{M}\right)=0$ if $\mu_{M}=0.075$ (that is to say, a gross expected return $m_{M}=1.095$ ). However, they do not exhibit a specific level of the market price $p_{M}$ nor they specify the exogenous supply $N$, the payoffs $\tilde{X}$ and the equilibrium prices $p$. We remark that equation $(7)$ is based on the return $\tilde{R}_{M}$. Therefore, we can exploit $(7)$ as long as we specify the parameters of $\tilde{R}_{M}$.

Conversely, suppose to know the parameters of the payoffs $\tilde{X}$ and the supply $N$. Now our goal is to find the equilibrium prices $p$. To fix ideas, we assume $J=1$ and $\tilde{X}_{1} \sim\left(m, s^{2}\right)$ for some $m \in \mathbb{R}$ and $s>0$. Let the exogenous supply of this security be $n_{1}>0$. In this case, $\tilde{M}=\tilde{X}_{1} n_{1}, m_{M}=m n_{1}$, $s_{M}^{2}=s^{2} n_{1}^{2}, p_{M}=p_{1} n_{1}$ and $\tilde{R}_{1}=\tilde{R}_{M}=\tilde{X}_{1} / p_{1}$. Consequently, the only price to be determined is the price $p_{1}$, or equivalently $p_{M}$. For a given price $p_{1}{ }^{3}$, we have

$$
\tilde{R}_{1} \sim N\left(\frac{m}{p_{1}}, \frac{s^{2}}{p_{1}^{2}}\right)
$$

which is well defined as long as $p_{1} \neq 0$. On the other hand, it seems reasonable to wonder what is the optimal demand of the investor when the risky asset price is zero. In other words, how many stocks is she willing to hold for free? Looking at (9), we see that $p_{1}$ tending to zero simultaneously drives the average return and its variance to infinity. However, $\tilde{R}_{1}$ does not converge in any natural sense and (7) is no more helpful. As in Del Vigna (2011), Proposition 2, we have the following result.

Proposition 2. Under Assumption 1-3, let $f$ be given by

$$
f\left(p_{1}\right)=\int_{0}^{+\infty} w(1-P(x)) \mathrm{d} v(x)-\int_{-\infty}^{0} w(P(x)) \mathrm{d} v(x),
$$

where the $c d f P$ is

$$
P(x)=\Phi\left(\frac{x+R_{f} p_{1}-m}{s}\right),
$$

and $\Phi$ is the cdf of a standard normal random variable. If $f(0) \leq 0$, then there are no CAPM equilibria.

Proof. Using Lemma 1 in Barberis and Huang (2008), we can compute

$$
V(\hat{W})=\theta_{1}^{\alpha} f\left(p_{1}\right)
$$

\footnotetext{
${ }^{3}$ Recall that our agents are price-taker.
} 
where $\theta_{1}$ is the number of risky securities owned by our investor ${ }^{4}$ and $f$ is given by (10). Equation (12) gives the optimal solution to the portfolio optimization problem:

$$
\theta_{1}= \begin{cases}0 & \text { if } f\left(p_{1}\right)<0 \\ \text { any } \theta_{1} \geq 0 & \text { if } f\left(p_{1}\right)=0 \\ +\infty & \text { if } f\left(p_{1}\right)>0\end{cases}
$$

Note that $f$ is continuous in $p_{1}$ and strictly decreasing. Moreover, $\lim _{p_{1} \rightarrow-\infty} f\left(p_{1}\right)=+\infty$ and $\lim _{p_{1} \rightarrow+\infty} f\left(p_{1}\right)=-\infty$. By the intermediate value theorem, there exists a unique $p_{1}^{\star} \in \mathbb{R}$ such that $f\left(p_{1}^{\star}\right)=0$. Hence, if $p_{1}^{\star} \leq 0$ then a CAPM equilibrium with positive prices does not exist.

We remark that equation (12) is based on a price and it is well defined even for $p_{1}=0$. Clearly, $p_{1}^{\star}$ is the equilibrium price as long as it is strictly positive. However, we can not a priori exclude $p_{1}^{\star} \leq 0$. Otherwise stated, even for an arbitrarily small (or zero) price, holding a positive amount of stocks is suboptimal for every agent. As a consequence, the market clearing condition can not be attained and no equilibrium with positive prices exists. Recall that also in expected utility agents models negative prices can arise (see Del Vigna (2011)). We interpret them as a sort of extreme reward for bearing risk or counterbalancing probable losses. We finally remark that the same reasoning applies in an economy with more than one risky asset. Furthermore, we could find a positive market price $p_{M}$ and at the same time some negative $p_{j}$, namely those of the riskier securities.

We now give a numerical example where the only attainable equilibrium implies a negative price for the risky asset. We recover the setting of the example by Barberis and Huang (2008), Section III.A. We arbitrarily fix $n_{1}$ and $m_{1}$ such that $m_{M}=1.095$. Leaving unchanged the other parameters, that is $\left(\alpha, \delta, \lambda, R_{f}\right)=(0.88,0.65,2.25,1.02)$, we can plot $f\left(p_{M}\right)$ for different values of $s_{M}$ (see Figure 1 ). The results are quite clear. An equilibrium exists as long as $s_{M}$ lies in a suitable range (numerical simulations show $s_{M} \in(0,2.19)$ ). Intuitively, this means that risk must be sufficiently low to attract positive risky investment.

A similar situation arises if we change the values of some preference parameters. We fixed $\left(\alpha, \delta, R_{f}, m_{M}, s_{M}\right)=(0.88,0.65,1.02,1.095,1.75)$ and allowed $\lambda$ to vary. Results are shown in Figure 2. In this case we can find an equilibrium only if $\lambda \in(1,2.77)$, i.e. if loss aversion is not too pronounced.

\section{References}

[1] N. Barberis and M. Huang (2008): Stocks as lotteries: The implications of probability weighting for security prices. The American Economic Review, 98(5), 2066-2100.

[2] E. G. De Giorgi, T. Hens and H. Levy (2011): CAPM equilibria with prospect theory preferences. Working Paper, available at http://ssrn.com/abstract $=420184$.

[3] M. Del Vigna (2011): Financial market equilibria with heterogenous agents: CAPM and market segmentation. Working Paper, available on request.

[4] A. Tversky and D. Kahneman (1992): Advances in prospect theory: Cumulative representation of uncertainty. Journal of Risk and Uncertainty, 5, 297-323.

\footnotetext{
${ }^{4}$ In equilibrium, the sum of $\theta_{1}$ over the set of agents must equal $n_{1}$ to clear the market.
} 


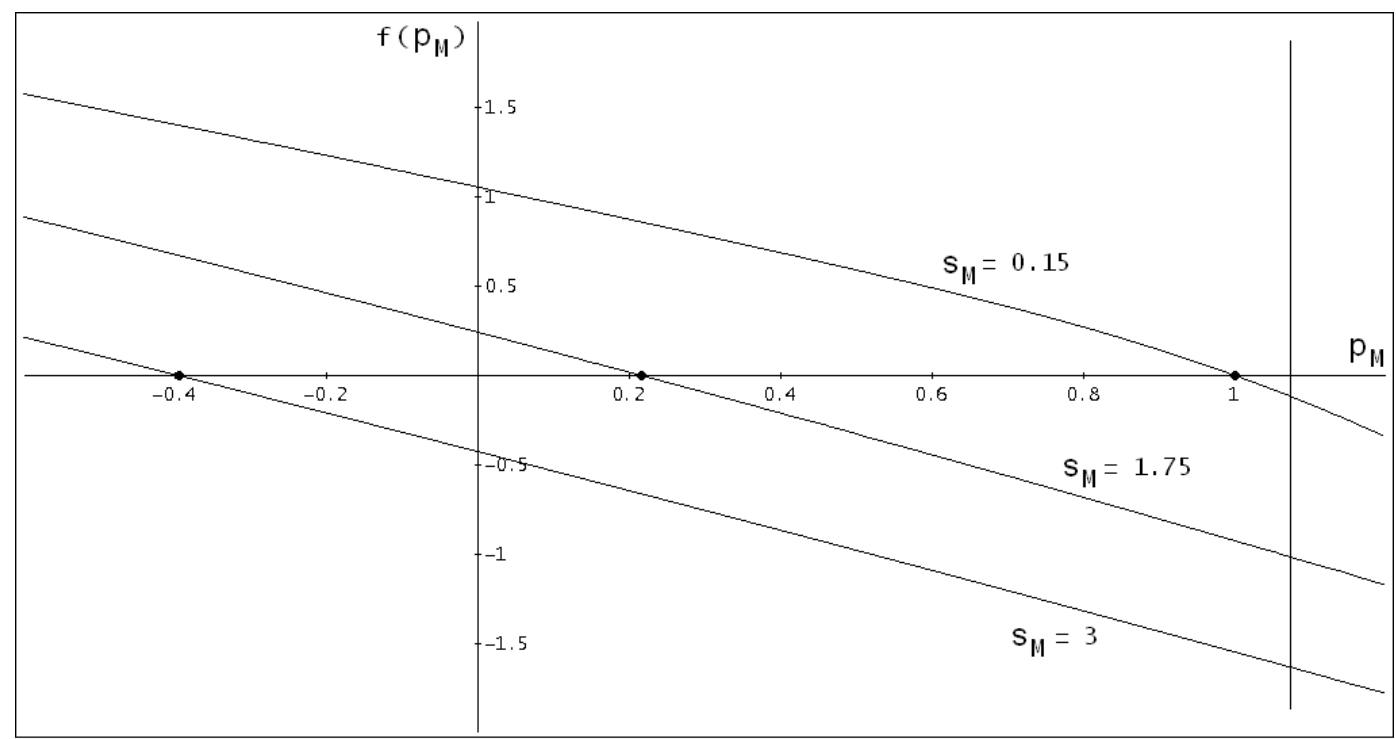

Figure 1: The figure shows three curves of the function $f$ introduced in (10). The preference parameters are $(\alpha, \delta, \lambda)=(0.88,0.65,2.25)$, the market parameters are $\left(R_{f}, m_{M}\right)=(1.02,1.095)$ and $s_{M}$ takes the values $0.15,1.75,3$. We highlighted with a dot the respective equilibrium prices: $p_{M}=1$ (as in the example of Barberis and Huang (2008)), $p_{M}=0.216$ and $p=-0.396$ (which must be refused). The vertical line represents the upper limit on $p_{M}$ given by (8), that is $p_{M}<1.095 / 1.02$.

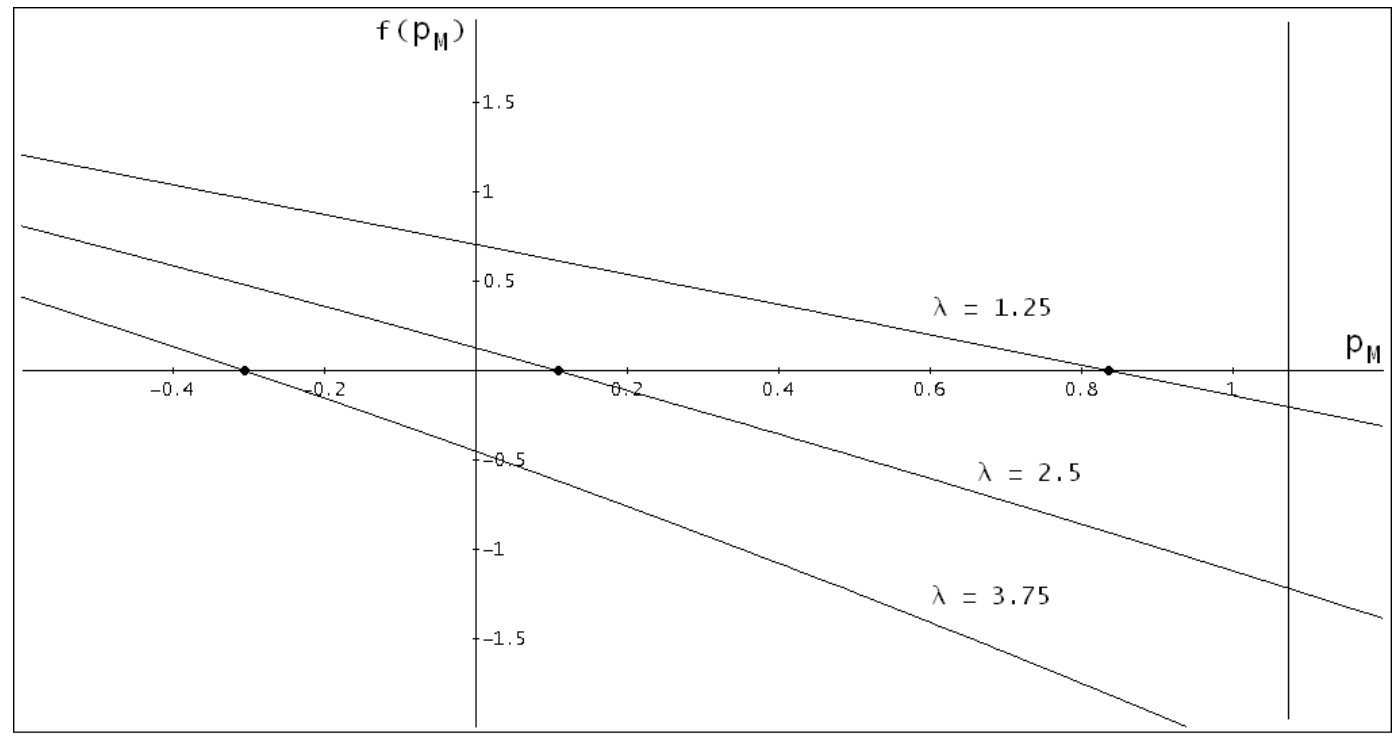

Figure 2: The figure shows three curves of the function $f$ introduced in (10). The market parameters are $\left(R_{f}, m_{M}, s_{M}\right)=(1.02,1.095,1.75)$, the preference parameters are $(\alpha, \delta)=(0.88,0.65)$ and $\lambda$ takes the values $1.25,2.5,3.75$. We highlighted with a dot the respective equilibrium prices: $p_{M}=0.836$, $p_{M}=0.107$ and $p_{M}=-0.306$ (which must be refused). The vertical line represents the upper limit on $p_{M}$ given by (8), that is $p_{M}<1.095 / 1.02$. 